\title{
FACTORS AFFECTING THE PENETRATION OF ANTIBIOTICS INTO THE AQUEOUS HUMOUR*
}

\author{
BY \\ MAURICE LANGHAM \\ Ophthalmological Research Unit, Medical Research Council, \\ Institute of Ophthalmology, London, W.C.1.
}

ESSENTIALLY the blood-aqueous barrier comprises the layers of cells which separate the blood of the capillary vessels within the globe of the eye from the intra-ocular fluids. Elucidation of its nature by dynamic studies has thrown considerable light on how substances circulating in the blood plasma pass into the aqueous humour. Water readily crosses all the cellular barriers bounding the anterior and posterior chambers of the eye at a much greater speed than substances dissolved in the plasma. The latter enter the eye at widely varying rates, but in general it has been found that substances readily soluble in fat solvents pass through the bloodaqueous barrier faster than those readily soluble in water; thus ethyl alcohol travels approximately five times as rapidly as the fastest water-soluble compound studied.

Consideration of the solubility characteristics of the chemotherapeutic substances recently introduced into clinical practice reveals that in general they are soluble in both water and lipids. The sulphonamides undergo ionization in the blood stream, and consequently exist as charged ions strongly soluble in water, and as uncharged molecules sparingly soluble in water but markedly soluble in ether and fats. The relative distribution of the two forms is such that the sulphonamides exist mainly in the uncharged form, soluble in fats. In similar manner, penicillin undergoes ionization in the blood stream and exists as water-soluble ions and as uncharged, undissociated fat-soluble molecules. Ionization of penicillin under these conditions is nearly complete, there being approximately one uncharged particle for every thousand ions. Thus sulphonamides in the blood stream exist predominantly as particles soluble in fats, whilst penicillin exists almost completely as ions insoluble in fats.

In the present work an attempt has been made to find a correlation between the rate of entry of the antibiotics into the aqueous humour and their physico-chemical character, and to investigate the premise that lipoid solubility is the main factor influencing speed of entry.

Received for publication July 5, 1951. 


\section{METHODS}

The following crystalline compounds were used*:

$p$-hydroxybenzyl penicillin $(\mathrm{X})$,

benzyl penicillin $(G)$,

2-pentenyl penicillin $(F)$,

$n$-amyl penicillin (dihydro $\mathrm{F}$ ),

$n$-heptyl penicillin $(\mathrm{K})$,

procaine benzyl penicillin (procaine penicillin $\mathrm{G}$ ),

3-( $\beta$-naphthylazo)- 4-hydroxybenzyl penicillin (naphthylazo penicillin X), chloromycetin.

Diffusion studies were carried out on conscious rabbits, a steady concentration of the antibiotic in the plasma being maintained by periodic intravenous injection. Paracentesis was performed under local anaesthesia (pantocaine solution). Experimental results were rejected if the aqueous humour contained significant amounts of protein.

Excluding chloromycetin, all estimations were carried out by the standard Oxford cup-plate technique, a control series being run alongside each series of determinations. Chloromycetin was estimated chemically by the Bratton-Marshall method for sulphonamides, being preceded by a reduction of the aromatic nitro group with zinc dust and dilute hydrochloride solution.

The degree of binding or adsorption on the plasma proteins was investigated with the dialysis chamber as described by Davson and others (1949a). In this apparatus, the blood plasma in a collodion sac is dialysed against isotonic sodium chloride solution. Estimation of the partition coefficient was made by dissolving the antibiotic in a $0.01 \mathrm{M}$ : phosphate buffer solution of $p \mathrm{H} 7.4$, adding ether and equilibrating on a shaker for two hours, and then estimating the concentration of the antibiotic in each phase.

\section{RESULTS}

The degree of binding of the compounds on some non-diffusible constituent of the plasma is recorded in Table I. Plasma containing the antibiotic was removed at the end of the experiment and dialysed against isotonic saline for 2 hours at room temperature. This length of time was found adequate for attainment of the equilibrium value; dialysis at room temperature instead of at blood temperature was found not to affect the degree of binding. In all cases the concentration of antibiotic in the

TABLE I

PERCENTAGE OF PENICILLIN BOUND TO PLASMA PROTEIN

\begin{tabular}{|c|c|c|c|c|c|c|}
\hline \multicolumn{4}{|c|}{ Antibiotic } & Arithmetic & S.E. & No. of \\
\hline $\begin{array}{l}\text { Penicillin } G \ldots \\
\text { Procaine penicillin } \ldots \\
\text { Dihydro penicillin } \mathrm{F} \\
\text { Penicillin K ... } \\
\text { Naphthylazo penicillin } \mathrm{X} \\
\text { Chloromycetin } \quad . .\end{array}$ & $\begin{array}{l}\cdots \\
\cdots \\
\cdots \\
\cdots \\
\cdots \\
\cdots\end{array}$ & $\begin{array}{l}\cdots \\
\cdots \\
\cdots \\
\cdots \\
\cdots \\
\cdots\end{array}$ & $\begin{array}{l}\cdots \\
\cdots \\
\cdots \\
\cdots \\
\cdots \\
\cdots\end{array}$ & $\begin{array}{r}50 \\
27 \\
75 \\
73 \\
100 \\
30\end{array}$ & $\begin{array}{l} \pm 1 \cdot 0 \\
\pm 4 \cdot 5 \\
\pm 2 \cdot 3 \\
\pm 3 \cdot 2 \\
\pm 2 \cdot 5\end{array}$ & $\begin{array}{l}4 \\
5 \\
3 \\
4\end{array}$ \\
\hline
\end{tabular}

*Samples of the natural penicillins were supplied by the courtesy of Boots Chemists, Nottingham, and the synthetic 3-( $\beta$-naphthylazo) - 4 - hydroxybenzyl penicillin by the Northern Regional Research Laboratories of America. 
plasma was found to be higher than in the saline. Naphthylazo penicillin was found to be completely adsorbed, this presumably being due to the presence of the azo group; likewise azo dyes are known to have a strong adsorption affinity for tissue proteins.

The relative speeds of penetration across the blood-aqueous barrier into the aqueous humour are expressed in Table II. With the concentration of the antibiotic in the plasma maintained approximately constant, the first sample of aqueous humour was withdrawn at between 20 and $30 \mathrm{~min}$. and the second at 40 to $60 \mathrm{~min}$. The constant of entry $(\mathrm{Ka})$ has been calculated according to the treatment of Davson and Quilliam (1940). The limitation of this equation to express the true rate of entry (Davson and others, 1949b) means that the recorded values must be considered as first approximations. Preliminary experiments showed that the normal aqueous humour contains no antibiotic; however, with chloromycetin it was considered advisable to remove the first sample of aqueous humour before beginning of the experiment for use as a blank.

TABLE II

RATES OF PENETRATION $\left(K_{i}^{*}\right)$ INTO AQUEOUS HUMOUR

\begin{tabular}{|c|c|c|c|c|c|c|}
\hline & & & & & & \\
\hline Antib & & & & $\begin{array}{l}\text { Arithmetic } \\
\text { mean }\end{array}$ & S.E. & $\begin{array}{c}\text { No. of } \\
\text { Experiments }\end{array}$ \\
\hline $\begin{array}{l}\text { Penicillin } G \ldots \\
\text { Procaine penicillin } \mathrm{G} \\
\text { Dihydro penicillin } \mathrm{F} \\
\text { Penicillin } \mathrm{K} \ldots \\
\text { Chloromycetin }\end{array}$ & $\begin{array}{l}\cdots \\
\cdots \\
\cdots \\
\cdots \\
\cdots\end{array}$ & $\begin{array}{l}\cdots \\
\cdots \\
\cdots \\
\cdots \\
\cdots\end{array}$ & $\begin{array}{l}\cdots \\
\cdots \\
\cdots \\
\cdots \\
\cdots\end{array}$ & $\begin{array}{l}3 \cdot 80 \\
4 \cdot 94 \\
5 \cdot 80 \\
12 \cdot 45 \\
68 \cdot 0\end{array}$ & $\begin{array}{l}-0 \cdot 3 \\
-0 \cdot 6 \\
-1 \cdot 1 \\
\div 1 \cdot 1 \\
\div 2 \cdot 0\end{array}$ & $\begin{array}{c}8 \\
7 \\
6 \\
10 \\
5\end{array}$ \\
\hline
\end{tabular}

* Permeability constant $K_{d} \quad 100 \log \left(\mathbf{S}-\mathbf{A}_{1}\right)-1 \quad \begin{aligned} & S \text { Mean plasma antibiotic concentration. } \\ & A_{1} \text {. Initial aqueous concentration at time } t\end{aligned}$ Time expressed in hours.

The results summarized in Table III show the relative affinity of the compounds studied for ether and phosphate buffer $(p \mathrm{H} 7.4)$. The substance dissolved in $0.1 \mathrm{M}$. phosphate buffer was shaken with ether for 3 hours at room temperature, and the concentration of substance in each phase measured. The higher values of the partition coefficient indicate a greater affinity of the substance for lipoid solvents.

TABLE III

THE ETHER WATER PARTITION COEFFICIENT IN 0.01 PHOSPHATE BUFFER $p \mathrm{H} 7 \cdot 4$

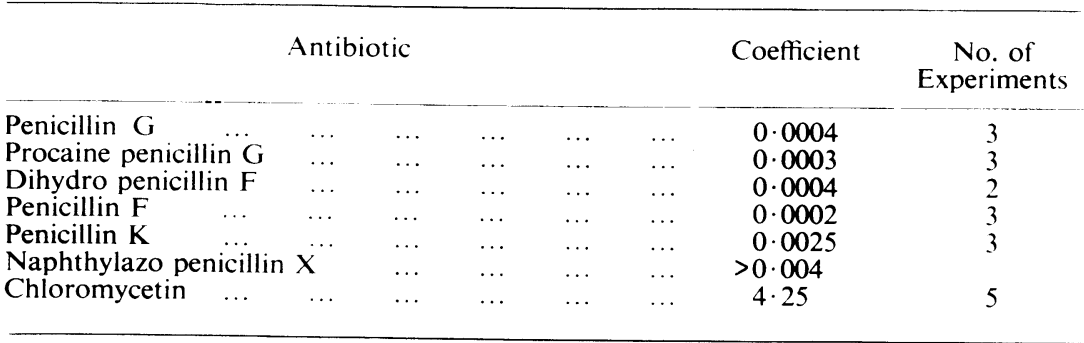


Discussion

The inability of substances soluble in water above a certain molecular size to penetrate into the aqueous humour has been used as experimental evidence to support the concept that the bloodaqueous barrier shows sieve-like properties. The disaccharide, sucrose (mol. wt. 342), travels across the blood-aqueous barrier approximately six times more slowly than the monosaccharides (mol. wt. 180), whilst raffinose, a trisaccharide (mol. wt. 504), is completely unable to pass across the barrier (Weld and others, 1942). The proteins of the plasma belong to this class of compounds, and because of their molecular size do not penetrate the blood-aqueous barrier. Adsorption of a substance on the proteins of the plasma will mean that it will not be able to penetrate the blood-aqueous barrier. In the present study, all the antibiotics have shown a marked affinity for the proteins of the plasma. In the case of the synthetic napthylazo penicillin, adsorption was found to be complete and none passed the blood-aqueous barrier into the aqueous humour. Thus it is obvious that the binding of the antibiotics to the proteins of the plasma, coupled with the fact that the blood-aqueous barrier is impermeable to these proteins, means that only the unbound fraction of the antibiotic in the bloodstream will be free to pass into the aqueous humour. Thus a knowledge of the degree of binding of a compound on the proteins of the plasma is essential to estimate the concentration of the compound in the plasma free to diffuse across the blood-aqueous barrier. In many abnormal conditions of the eye, the restraining influence of the barrier to the passage of proteins breaks down, and in consequence the amount of antibiotic free to pass across the barrier is likely to increase. This was seen in the present work where the secondary plasmoid aqueous humour, formed after removal of the normal protein-free sample of the aqueous humour, was found to contain a concentration of antibiotic equal to that in the plasma, but still bound to protein.

Owing to a drainage in bulk of the aqueous humour from the eye, it is found that substances which accumulate in the aqueous humour below a certain speed never reach a concentration equal to that in the plasma. This is exemplified by urea, whose concentration in the aqueous humour at the time of equilibrium is only 75 per cent. of that in the plasma. An equilibrium of this nature is referred to as a "steady-state". For convenience, this value is generally referred to as a steady-state ratio, denoted by $R$ eq, which may be defined as the ratio of the concentrations of a substance in the aqueous humour and the plasma water when the concentration in the aqueous humour is no longer rising. This type of dynamic equilibrium is in marked contrast to what occurs in non-living systems. Thus if a membrane separates two concentrations of a substance in solution to which the membrane is permeable, this substance will travel across 
the membrane until the concentrations on either side are equal; chemical equilibrium is then said to exist. Excluding chloromycetin, all the substances studied diffuse across the blood-aqueous barrier more slowly than urea, and in consequence their concentration in the aqueous humour will always be less than 75 per cent. of that found free in the plasma. Chloromycetin, however, passes in faster than urea and in consequence its steady-state will be greater than 0.75 . The maximum steady-state ratio for a non-electrolyte that enters by diffusion is unity; that is, the concentration in the aqueous humour will equal the concentration in the plasma. In the case of chloromycetin, the ratio will certainly be nearer unity than 0.75 for it travels across approximately five times faster than urea. This conclusion is supported by the finding that methyl thiourea which enters the aqueous humour slightly more slowly than chloromycetin has a steady-state ratio of 0.95 (Ross, 1951).

The partition coefficient expresses the distribution of a substance between two immiscible solvents, e.g., oil and water. A substance soluble in oil only will dissolve in the oil phase when added to a mixture of oil and water; likewise an oil-insoluble compound like glucose will remain completely in the aqueous phase. It follows that the partition coefficients of substances strongly soluble in water will be low, e.g., glucose 0.0000045 , sucrose 0.0000011 , and $\alpha$-alanine 0.000014 , whilst for strongly lipoid substances the values will be high, e.g., alcohol 0.26 and ether 10 (Collander, 1949). The compounds studied in the present work have characteristics which place them between these two extremes. The relation between the rates of entry and the value of the partition coefficient is summarized in the Figure. Three results have been taken from the work of Davson and others (1949) to give a more representative idea of the general relationship. An increase in the value of the partition coefficient indicates an increased lipoid solubility. It will be noted that there is a general relationship between the rate of entry and the lipoid solubility of the compound. Penicillin, which exists in the plasma in the strongly water-soluble ionic form, penetrates the bloodaqueous barrier very slowly; in contrast, chloromycetin which is more soluble in fat solvents passes very readily across the barrier. Investigating a related series of non-electrolytes, Ross (1951) has similarly demonstrated a direct relationship between the rate of entry into the aqueous humour and lipoid solubility.

Further consideration of the Figure suggests that the molecular weight, or size, of these compounds has no marked effect on their rate of entry. Chloromycetin travels across the barrier approximately twenty times more rapidly than penicillin $G$, yet these two compounds have similar molecular weights (penicillin G 334, and chloromycetin 335). This supports the view that it is the relative 
affinity of these compounds for the lipids of the cell membranes which determines their rate of entry. When we compare penicillin $\mathrm{K}$ with dihydro penicillin $F$, we find that, though the molecular weight of penicillin $K$ (342) is appreciably higher than that of dihydro penicillin $F(312)$, penicillin $\mathrm{K}$ passes through the barrier two or three times faster.

These present results support the concept that the blood-aqueous barrier has both sieve and lipoid properties. It would seem probable that compounds strongly soluble in water, such as sugars and amino acids, which

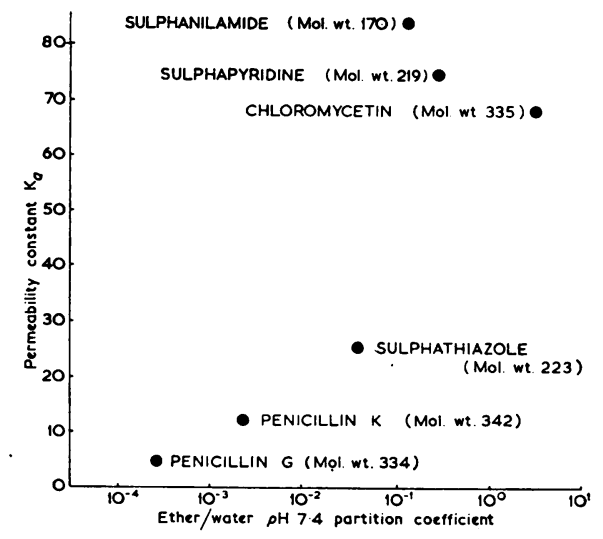

FIGURE. - Permeability of blood-aqueous barrier to various antibiotics.

Ordinate: rate of penetration into aqueous humour expressed as permeability constant $\mathrm{K}_{a}$.

Abscissa: lipoid solubility expressed as ether/water $p H$ 7.4 partition coefficient.

(Sulphonamide values from Davson and others, 1949) have partition coefficients so low that for practical purposes they may be considered completely lipoid-insoluble, enter through intercellular spaces. The main evidence for this is the fact that the rate of entry is in inverse proportion to the size of the particle; the size of the holes is shown by the fact that sucrose (mol. wt. 342) does pass into the anterior chamber whereas raffinose (mol. wt. 504) does not. In contrast, in the case of lipoid-soluble compounds, the present evidence indicates that size can be of only secondary importance, the rate of entry being proportional to the lipoid solubility of the compound. Results in the present study suggest that benzyl penicillin represents a compound whose physical characteristics place it on an arbitrary line dividing the two classes of compounds. It has a partition coefficient which is 10 to 100 times higher than that of the sugars and simple amino acids like $\alpha$-alanine, but is still 10,000 times less than that of the strongly lipoid-soluble chloromycetin. It is suggested, therefore, that the effect of lipoid solubility on rate of entry becomes apparent for those compounds having partition coefficients greater than approximately 0.0004 .

Certain of the antibiotics recently introduced into general therapy have physical characteristics which indicate that they will pass the blood-aqueous barrier at very low speeds. Streptomycin is a lipoidinsoluble compound; therefore its entry will depend on its molecular size. Now it will be remembered that the limit of size of particles for a substance entering via the cellular spaces by simple diffusion is of the order of a mol. wt. of 500; streptomycin, having a mol. wt. of 743 , would not be expected to pass the barrier. This was confirmed 
experimentally in the case of rabbits. Aureomycin and polymyxin also are both compounds of a lipoid-insoluble nature and would not be expected to cross the barrier rapidly.

If the concept of the barrier having both sieve and lipoid characteristics is accepted, the differences in rates of penetration between the substances entering solely via intercellular spaces and those entering across cell membranes is more easily explained. Whilst lipoid-soluble substances may enter through all the barriers bounding the aqueous humour, the presence of intercellular spaces is likely to be restricted mainly to the anterior surface of the iris, where there is only a thin incomplete layer of endothelium separating the aqueous humour from the blood stream. This view is supported by the results of Davson and Duke-Elder (1948) on the rate of diffusion of sugars across the blood-aqueous barrier. These workers found that rate of penetration tended to increase with a decrease in the size of the pupil, i.e., with an increase of the exposed area of the iris. In contrast, in the region of the ciliary body where active transfer of substances into the aqueous humour is known to occur, the barrier consists of the capillary endothelium, the lamina vitrea, and a double layer of epithelial cells, and here the likelihood of the barrier having sieve properties is small.

\section{SUMMARY}

(1) Determination of the rates of entry, the degree of adsorption on the proteins of the plasma, and the value of the ether/phosphate $(p \mathrm{H}$ 7.4) partition of various antibiotics has been made.

(2) Results indicate that the rate of entry of the compounds may be related directly to their lipoid solubility as expressed by the

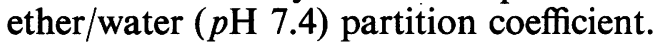

(3) The maximum concentration of an antibiotic in the aqueous humour of a normal eye for a given concentration in the blood stream is dependent not only on the rate of entry but also on its degree of adsorption on the proteins of the plasma.

(4) Of the antibiotics in common clinical use, chloromycetin (chloramphenicol) passes the most readily into the intra-ocular fluids from the blood stream, so that with this method of administration a high concentration of the drug within the eye is readily attained.

I should like to thank Sir Stewart Duke-Elder and Dr. H. Davson for their interest in this work.

\section{REFERENCES}

Bratton, A., and Marshall, E. K. JNR. (1939). J. biol. Chem., 128, 537.

Collander, R. (1949). Acta chemica scand., 3, 717.

Davson, H. (1950). J. Physiol., Lond., 110, 416.

and DuKe-Elder, W. S. (1948). Ibid., 107, 141.

and Maurice, D. M. (1949a). İbid., 109, 32

Ross, E. J., and Woodin, A. M. (1949b). Ibid., 108, 203.

and Quilliam, J. P. (1940). Ibid., 98, 141 .

Ross, E. J. (1951). Ibid., 112, 229.

Whi D, C. B., Feindel, W. H., and Davson, H. (1942). Amer. J. Physiol., 137, 421. 